\title{
The Adaptive Chaotic Symbiotic Organisms Search Algorithm Proposal for Optimal Reactive Power Dispatch Problem in Power Systems
}

\author{
Enes Yalçın' ${ }^{1}$, M. Cengiz Taplamacıoğlu' ${ }^{1 D}$, Ertuğrul Çam² \\ 'Department of Electrical and Electronics Engineering, Gazi University, Ankara, Turkey \\ 2Department of Electrical and Electronics Engineering, Kırıkkale University, Kırıkkale, Turkey
}

Cite this article as: Yalçın E, Taplamacıoğlu MC, Çam E." The Adaptive Chaotic Symbiotic Organisms Search Algorithm Proposal for Optimal Reactive Power Dispatch Problem in Power Systems", Electrica, 2019; 19(1): 37-47.

\begin{abstract}
This paper presents an adaptive chaotic symbiotic organisms search algorithm (A-CSOS) for finding the solution of optimal reactive power dispatch (ORPD) problem which is one of the main issues of power system planning and operations. The most important advantage of symbiotic organisms search algorithm (SOS) is that is not need any particular algorithm parameters. However, the SOS algorithm has some features to be enhanced, like falling into local minima and sluggish convergence. A-CSOS algorithm with adding new and improved features like adaptivity and chaos to conventional SOS algorithm is proposed to solve ORPD problem. The ORPD problem is mainly focused on minimization of transmission loss (Ploss) and total voltage deviation (TVD). To determine the optimal set points of control variables including generator bus voltages, tap positions of transformers, and reactive power outputs of shunt VAR compensators is very crucial for minimization to Ploss and TVD. The proposed algorithm is implemented on IEEE 30-bus test power systems for ascertaining the performance of A-CSOS algorithm on ORPD problem. The results showed that the proposed approach is up to $10.39 \%$ better than many of which the latest algorithms in literature and encourage the researchers to implement A-CSOS algorithm to ORPD problem. Keywords: Adaptive chaotic symbiotic organisms search, power loss minimization, reactive power dispatch, symbiotic organisms search, voltage deviation minimization
\end{abstract}

\section{Corresponding Author: \\ Enes Yalçın}

E-mail:

gaziyalcine@gmail.com

Received: 16.07 .2018

Accepted: 26.11 .2018

(c) Copyright 2019 by Electrica Available online at

http://electrica.istanbul.edu.tr

DOI: 10.26650/electrica.2019.18008

\section{Introduction}

Efforts to find the optimum solution for power system planning and operational problems continue today. One of these problems is the optimal reactive power dispatch (ORPD) problem which is a highly nonlinear and non-convex optimization problem [1]. The ORPD can be defined as an ideal allocation of reactive power in the power system to minimize predefined objective function while satisfying the numerous constraints. Though active power loss (Ploss) is mostly preferred as an objective function to be minimized, minimization of absolute value of total voltage deviation (TVD) and voltage stability index can be used as an objective functions in ORPD studies. Therefore, minimization of Ploss and TVD have been chosen as objective functions in this study. The parameters that are controlled to minimize the objective function are generator bus voltages, tap settings of transformers and reactive power outputs of shunt compensators [2].

Up to now, many algorithms from classical optimization techniques to modern optimization and hybrid algorithms have been used to determine the ideal values of the control parameters. Many different modern optimization techniques such as particle swarm optimization (PSO) [3], differential evalution (DE) [4], biogeography based optimization (BBO) [5], gravitational search algorithm (GSA) [6], artificial bee colony (ABC) [7], firefly algorithm (FA) [7], bacteria foraging optimization algorithm (BFOA) [7], bat algorithm (BA) [8], cuckoo search algorithm (CSA) [8], ant lion optimization (ALO) [9], gray wolf optimization (GWO) [9], teaching learning based optimization (TLBO) [10], whale optimization algorithm (WOA) [11], quasi-oppositional chemical reaction optimization (QOCRO) [12] are being developed and applied to ORPD and other optimization problems. However, these algorithms also have features that can be positive and negative or improved. For this reason, existing algorithms continue to 
be improved either by hybridization with more than one algorithm or by adding various features to the existing algorithm. The comprehensive learning PSO (CLPSO) [3], hybrid particle swarm optimization and gravitational search algorithm (PSOGSA) [6], opposition-based gravitational search algorithm (OGSA) [13], hybrid firefly algorithm (HFA) [7], modified ant lion optimization (MALO) [8], quasi-oppositional teaching learning based optimization (QOTLBO) [10], modified differential evolution (MDE) [14], improved gravitational search algorithm with conditional selection strategies (IGSA-CSS) [15], chaotic improved particle swarm optimization (MOCIPSO) [16], chaotic parallel vector evaluated interactive honey bee mating optimization (CPVEIHBMO) [17], hybrid particle swarm optimization and imperialist competitive algorithm (PSO-ICA) [18] can be given as example of algorithms developed with this approach for solving the ORPD problem. However, most of these modern optimization algorithms contain some parameters that must be determined sensitively and affecting the result significantly.

As a solution to this problem, Symbiotic Organisms Search algorithm (SOS) is introduced [19]. SOS is an algorithm that is inspired by the interaction of the organisms in an ecosystem and does not contain any particular algorithmic parameters. SOS algorithm has been implemented on some power system problems such as economic load dispatch by Guvenc et al. [20], optimal placement of distributed generations in radial distribution systems by Das et al. [21. However, SOS algorithm offers many important advantages but also SOS may suffer from premature convergence that will lead the optimization falling into local optima when it is applied for high dimension large-scale problems. For this reason, some researchers such as Secui [22] and Saha et al. [23] have achieved better results than standard SOS by making some modifications based on the standard SOS. To find a better result in global solution set by improving searching capability and avoid falling into local optima, principle of chaos approach adapted to algorithms. The making some modifications and using hybridization techniques affect positively to performance of originals. However, handling the constraints within limits cannot be assured when using these methods to solve especially complex optimization problems. To find global optimum solution for large-scale problems like ORPD problem, not only to improve the original methods but also to handle constraints have to be required well simultaneously. In many studies, quadratic penalty function is used to overcome all equality and inequality constraints, but this method has some penalty parameters that significantly affect the solution and to be needed a large amount of time to determine the optimal values. In addition to this, static penalty method also has some coefficients that significantly affect the solution and be required time-consuming trial and errors. More recently, adaptive penalty schemes have been introduced with the goal of eliminating above-mentioned problems and evaluating each candidate solution using specific feedback information for every iteration. One of the most promising self-adaptive penalty approach is the Global Competitive Ranking (GCR) method [24].
In this paper, adaptive chaotic symbiotic organisms search algorithm (A-CSOS) is designed by integrating the chaos and adaptive penalty features into SOS. The proposed A-CSOS algorithm is applied to ORPD problem comprising the Ploss and TVD minimization on IEEE 30-bus test power system. Simulations are performed on four different test cases which are Ploss minimization with continuous variables, Ploss minimization with discrete variable transformer taps and shunt compensator outputs, TVD minimization with continuous variables, TVD minimization with discrete variable transformer taps and shunt compensator outputs. Simulation results show that the proposed algorithm gives substantially better result than the best result of many other state-of-art algorithms. Therefore, A-CSOS will be one of the most promising algorithm for ORPD and an encouraging algorithm for other constrained optimization problems.

\section{Problem Description}

The minimization of Ploss which is the first objective function in this study, means the amount of active power losses in transmission lines. TVD minimization, which is taken as the second objective function in this study, is used for minimizing absolute deviations of all the actual PQ bus voltages from their desired or set values. The value of set voltage $\left(V_{i}^{\text {set }}\right)$ is considered 1.0 p.u. for TVD minimization. The formulation of Ploss and TVD minimization are shown in Eq. (1) and Eq. (2), respectively.

$$
\begin{gathered}
f_{1}\left(x_{1}, x_{2}\right)=\sum_{b r=1}^{N_{b r}}\left[G _ { b r } \left(V_{i}^{2}+V_{j}^{2}\right.\right. \\
\left.\left.-2 V_{i} V_{j} \cos \delta_{i j}\right)\right] \\
f_{2}\left(x_{1}, x_{2}\right) \\
=\sum_{l=1}^{N_{P Q}}\left|V_{l}-V_{l}^{\text {set }}\right|
\end{gathered}
$$

In Eq. (1) and (2), $f_{1}$ and $f_{2}$ denote to first and second objective functions, respectively; $x_{1}$ and $x_{2}$ denote to dependent and independent variables, respectively; $N_{b r}$ express the total number of branches; $G_{b r}$ is the conductance of line-br connecting buses $i$ and $j ; V_{i}$ is the bus voltage magnitude at bus $i ; \delta_{i j}$ is the phase angle difference between bus- $i$ and $j ; N_{P Q}$ is the number of PQ-bus.

Subject to equality and inequality constraints are represented by Eq. (3) and Eq. (4), respectively.

$$
\begin{aligned}
& g\left(x_{1}, x_{2}\right)=0 \\
& h\left(x_{1}, x_{2}\right) \leq 0
\end{aligned}
$$

The vector of state variable, $x_{1}$ shown in Eq. (5), compose of load bus voltages $\left(V_{1}\right)$, generators' Mvar outputs $\left(Q_{G}\right)$ and line $x_{1}$

$=\left[V_{l 1}, \ldots, V_{l N_{P Q}}, Q_{G 1}, \ldots, Q_{G N_{P V}}, S_{b r 1}, \ldots, S_{b r N_{b r}}\right]$ 
$x_{2}$, shown in Eq. (6), represents the control variables including bus voltage magnitudes of PV bus $\left(V_{G}\right)$, tap ratios of transformers $(T)$ and Mvar output of shunt compensators $\left(Q_{c}\right)$, respectively.

$$
\begin{aligned}
& x_{2} \\
& =\left[V_{G 1}, \ldots, V_{G N_{P V}}, T_{1}, \ldots, T_{N_{T}}, Q_{c 1}, \ldots, Q_{c N_{c}}\right]
\end{aligned}
$$

where $N_{P V}$ is the number of PV-bus, $N_{T}$ is the number of tap changing transformers, $\mathrm{N}_{\mathrm{c}}$ is the number of VAR compensators.

While both two objective functions are minimized, all equality and inequality constraints must be satisfied simultaneously.

The equality constraints denoted by $g$ in Eq. (3) are shown in Eq. (7) and (8).

$$
\begin{aligned}
& P_{G_{i}}-P_{L_{i}}-V_{i} \sum_{j=1}^{N_{B}} V_{j}\left[G_{i j} \cos \left(\delta_{i}-\delta_{j}\right)\right. \\
& \left.+B_{i j} \sin \left(\delta_{i}-\delta_{j}\right)\right]=0, \quad i \\
& \in N_{P Q} \\
& Q_{G_{i}}-Q_{L_{i}}-V_{i} \sum_{j=1}^{N_{B}} V_{j}\left[G_{i j} \sin \left(\delta_{i}-\delta_{j}\right)\right. \\
& \left.-B_{i j} \cos \left(\delta_{i}-\delta_{j}\right)\right]=0, \quad i \\
& \in N_{P Q}
\end{aligned}
$$

where $N_{B}$ is the number of bus, $P_{G i}$ and $Q_{G i}$ are the amount of of active and reactive power generation for bus $i$, respectively; $P_{L i}$ and $Q_{L i}$ are the amount of of active and reactive power load for bus $i$, respectively.

The inequality constraints denoted by $h$ in Eq. (4) are composed of the maximum and minimum limits of generator and load bus voltages, the minimum and maximum reactive power outputs of generators and shunt compensators, the minimum and maximum ratios of tap changing transformers, the maximum line capacity expressed in Eq. (9-14), respectively.

$$
\begin{aligned}
& V_{G i}^{\min } \leq V_{G i} \leq V_{G i}^{\max }, i=1,2, \ldots, N_{G e n} \\
& V_{l i}^{\text {min }} \leq V_{l i} \leq V_{l i}^{\max }, i=1,2, \ldots, N_{P Q} \\
& Q_{G e n i}^{\min } \leq Q_{G e n i} \leq Q_{G e n i}^{\max }, i=1,2, \ldots, N_{G e n} \\
& Q_{C i}^{\min } \leq Q_{C i} \leq Q_{C i}^{\max }, i=1,2, \ldots, N_{C} \\
& T_{i}^{\min } \leq T_{i} \leq T_{i}^{\max }, \quad i=1,2, \ldots, N_{T} \\
& \left|S_{b r_{i}}\right| \leq S_{b r_{i}}^{\max }, i=1,2, \ldots, N_{b r}
\end{aligned}
$$

\section{Methods}

The SOS algorithm has been developed as an important alternative to algorithms that have some algorithmic parameters that affect solution accuracy significantly and that must be specified by the user. Due to this advantage, it has been applied to many optimization problems to date and successful results have been obtained.

However, as the problem of applying the SOS algorithm becomes more complicated, the convergence time of the algorithm is prolonged and the accuracy of solution is insufficient. Therefore, the exploration and exploitation capabilities, and convergence speed of standard SOS should be enhanced.

In this section, the standard SOS algorithm is briefly explained, and then the modifications on the SOS are described in the subheadings.

\section{SOS Algorithm}

Symbiotic Organisms Search algorithm inspired by the symbiotic interactions between different organisms living in an ecosystem [19]. The mutualism, commensalism, and parasitism constitute the basic relations of SOS.

\section{Mutualism:}

Mutualism is a relationship based on the fact that the two organism in the ecosystem benefit more or less from one another. The relationship between bee and flower is an example of this phenomenon. Not only bees get benefited by collecting nectar from flower for producing into honey, but also flowers get benefited from bees that help to flowers to become fruit by pollination. This phase is mathematically expressed as follows in the standard SOS.

$$
\begin{aligned}
& X_{i}^{\text {new }}=X_{i}^{a c t}+\operatorname{rand} \times\left(X_{\text {best }}-\frac{X_{i}^{a c t}+X_{j}^{a c t}}{2} \times B F_{1}\right) \\
& X_{j}^{\text {new }}=X_{j}^{a c t}+\operatorname{rand} \times\left(X_{\text {best }}-\frac{X_{i}^{a c t}+X_{j}^{a c t}}{2} \times B F_{2}\right) \\
& B F_{1}=\operatorname{round}(1+\text { rand }) \\
& B F_{2}=\operatorname{round}(1+\operatorname{rand})
\end{aligned}
$$

In above equations, $X_{i}^{\text {act }}$ represents an organism that corresponds to $i$ th organism in the ecosystem, $X_{j}^{\text {act }}$ represents a randomly selected organism that interacts with $X_{i}^{a c t} ; X_{\text {best }}$ represents an organism with the minimum fitness value in the ecosystem; $X_{i}^{\text {new }}$ and $X_{j}^{\text {new }}$ denote the new obtained organisms after performing mutualism; the rand expression is a random value between 0 and $1 ; B F_{1}$ and $B F_{2}$ are benefit factors which represent the level of benefit to each organism. If the value of $B F$ is 1 , organisms are benefitted partially; otherwise one organism is benefited fully from this relationship. The new obtained organisms $X_{i}^{\text {new }}$ and $X_{j}^{\text {new }}$ are compared with $X_{i}^{\text {act }}$ and $X_{j}^{\text {act }}$ according to their fitness value and then the organisms that have better fitness value are accepted. 


\section{Commensalism:}

Commensalism is the type of relationship in which one organism in the ecosystem benefits from this relation while the other is unaffected. The relationship between shark and remora fish is an example of this phenomenon. Remora fish adhere to the shark and feeds by eating residue from shark's food. Therefore, the remora fish get benefit, whereas the shark is not affected by the natural process of remora fish. This phase is mathematically expressed as follows in standard SOS:

$$
X_{i}^{\text {new }}=X_{i}^{a c t}+\operatorname{rand}(-1,1)\left(X_{\text {best }}-X_{j}^{a c t}\right)
$$

where $X_{i}^{\text {new }}$ denotes the new obtained organism after performing commensalism; the expression $\operatorname{rand}(-1,1)$ is the random value between -1 and 1 . The assessment of the obtained new organism $X_{i}^{\text {new }}$ is the same as in the mutualism phase.

\section{Parasitism:}

Parasitism is defined as the type of relationship that one of the two organisms in the ecosystem has benefited from this relation while the other is harmed. The interaction between a plasmodium anopheles and human is an example of this phenomenon. If an infected anopheles mosquito bites at human, anopheles gets benefit because of feeding. The human, by contrast, is damaged by a fatal parasite that causes malaria.

$X_{i}^{a c t}$ denotes to parasite vector. To be a parasite vector, modifies itself by the help of a random vector. For hosting to the parasite vector, an organism $X_{j}^{a c t}$ is randomly selected. The fitness value of infected vector $X_{j}^{a c t}$ and parasite vector $X_{i}^{a c t}$ is calculated. If the parasite vector has a better fitness value than infected vector, kills to infected vector and substitute $X_{j}^{a c t}$, otherwise, $X_{j}^{a c t}$ kills to parasite vector and remain the position of $X_{j}^{a c t}$.

\section{A-CSOS Algorithm}

Symbiotic Organisms Search algorithms has some disadvantages such as slow convergence and falling into local optima. Therefore, the global and local searching abilities, and convergence capability of standard SOS can be enhanced.

Chaos maps are known to significantly increase the exploration and exploitation proficiencies of the algorithms. On the other hand, the success of an algorithm depends not only on its own capabilities but also on the chosen constraint handling strategy. Standard SOS is enhanced by incorporating the chaos and adaptive penalty features. The modifications are specified in following subsections.

\section{Chaos Integration in Mutualism and Commensalism Phases}

In this study, logistic map that is one of the most used chaotic maps is preferred. The logistic map is applied to the rand statement in mutualism phase and rand $(-1,1)$ statement in commensalism phase which is expressed in state equation form as:

$$
c_{i, j}^{k}=4 c_{i, j-1}^{k}\left(1-c_{i, j-1}^{k}\right)
$$

where $c_{i j}^{k}$ represents the $j$ th chaotic variable of $i$ th individual in the $k$ th iteration; initial value of chaotic variable $C_{0}$ can take any value between 0 and 1 except $0.25,0.5$, and 0.75 . The updated formulas of the mutualism and commensalism phases after the modifications are shown in Eq. (21-23).

$$
\begin{aligned}
& X_{i}^{\text {new }}=X_{i}^{a c t}+c_{i}^{k}\left(X_{\text {best }}-\frac{X_{i}^{a c t}+X_{j}^{a c t}}{2} \times B F_{1}\right) \\
& X_{j}^{\text {new }}=X_{j}^{a c t}+c_{j}^{k}\left(X_{\text {best }}-\frac{X_{i}^{a c t}+X_{j}^{a c t}}{2} \times B F_{2}\right) \\
& X_{i}^{\text {new }}=X_{i}^{a c t}+\left(2 c_{i}^{k}-1\right)\left(X_{\text {best }}-X_{j}^{a c t}\right)
\end{aligned}
$$

\section{Global Compatitive Ranking}

In most studies, if one of the constraints is exceeded, a penalty value is added to the objective function for manipulating candidate individual from infeasible to feasible region. Commonly used fitness function for ORPD problem is given as follow:

$$
\begin{gathered}
F\left(X_{i}\right)=f\left(X_{i}\right)+k_{v} \sum_{i=1}^{N_{P Q}}\left(V_{l_{i}}-V_{l_{i}}^{\text {lim }}\right)^{2} \\
+k_{Q} \sum_{i=1}^{N_{G e n}}\left(Q_{G e n_{i}}\right. \\
\left.-Q_{G e n_{i}}^{\text {lim }}\right)^{2}
\end{gathered}
$$

where $f\left(X_{i}\right)$ denotes the objective function value of or organism-i; $k_{V}$ and $k_{Q}$ are expressed as static penalty factors; $V_{l i}^{\lim }$ and $V_{\text {Gini }}$ are the permissible limits of load bus voltage and Mvar output of generators, respectively. These limits are considered as follows:

$$
\begin{aligned}
& V_{l_{i}}^{\text {lim }}=\left\{\begin{array}{c}
V_{l_{i}}^{\min }, \text { if } V_{l_{i}}<V_{l_{i}}^{\min } \\
V_{l_{i}}^{\text {max }}, \text { if } V_{l_{i}}>V_{l_{i}}^{\max } \\
V_{l_{i}}, \text { if } V_{l_{i}}^{\text {min }} \leq V_{l_{i}} \leq V_{l_{i}}^{\max }
\end{array}\right. \\
& Q_{G e n_{i}}^{\lim }=\left\{\begin{array}{c}
Q_{G e n_{i}}^{\min }, \text { if } Q_{G_{G e n_{i}}}<Q_{G e n_{i}}^{\min } \\
Q_{G e n_{i}}^{\max }, \text { if } Q_{G e n_{i}}>Q_{G e n_{i}}^{\max } \\
Q_{G_{\text {Gen }}}, \text { if } Q_{G e n_{i}}^{\min } \leq Q_{\text {Gen }_{i}} \leq Q_{\text {Gen }_{i}}^{\max }
\end{array}\right.
\end{aligned}
$$

It is very difficult and time-consuming process to determine ideal values of the penalty parameters in static penalty method. Moreover, the solution is very sensitive to penalty coefficients. The mentioned drawbacks of the static penalty function blight the performance of the SOS algorithm. For this reason, Global Competitive Ranking has an important advantage with this aspect. 
One of the adaptive penalty methods is Global Competitive Ranking (GCR) developed by Runarsson and Yao. GCR is a ranking-based constraint handling method and strikes a balance between objective function and the sum of constraints violations using the following expressions [24]

$$
\begin{aligned}
F\left(X_{i}\right)=P_{f} \frac{\operatorname{rank}_{1}-1}{N-1} & \\
& +(1 \\
& \left.-P_{f}\right) \frac{\operatorname{rank}_{2}-1}{N-1}
\end{aligned}
$$

where

$$
\begin{aligned}
& \operatorname{rank}_{1}=\operatorname{rank}\left(f\left(X_{i}\right)\right) \\
& \operatorname{rank}_{2} \\
& =\operatorname{rank}\left(\sum_{j=1}^{m} v_{j}\left(X_{i}\right)\right) \\
& v_{j}\left(X_{i}\right)=\max \left\{0, g_{j}\left(X_{i}\right)\right\}
\end{aligned}
$$

In above equations, $N$ is the number of candidate solution in the population; $\operatorname{rank}\left(f\left(X_{j}\right)\right)$ and $\operatorname{rank}\left(\sum_{j=1}^{m} v_{j}\left(X_{j}\right)\right)$ denote the current ranking position of the candidate solution $X_{i}$ based on its objective function value and the sum of its constraint violations, respectively; $v_{j}\left(X_{j}\right)$ is the amount of violation of the $j$-th constraint for the candidate solution $X_{i} ; P_{f}$ represents the probability that an individual's fitness function value is determined according to its objective function value. It is suggested by the author that a value between 0 and 0.5 for the $P_{f}$ value.

The flow diagram for solving the ORPD problem with the A-CSOS algorithm is shown in Figure 1.

\section{Findings}

Optimal reactive power dispatch problem is applied on IEEE 30-bus system. Within this scope, the following four different cases are studied in this paper.

Case-1: Ploss minimization with continuous variables

Case-2: Ploss minimization with discrete variable transformer taps and shunt compensator outputs

Case-3:TVD minimization with continuous variables

Case-4: TVD minimization with discrete variable transformer taps and shunt compensator outputs

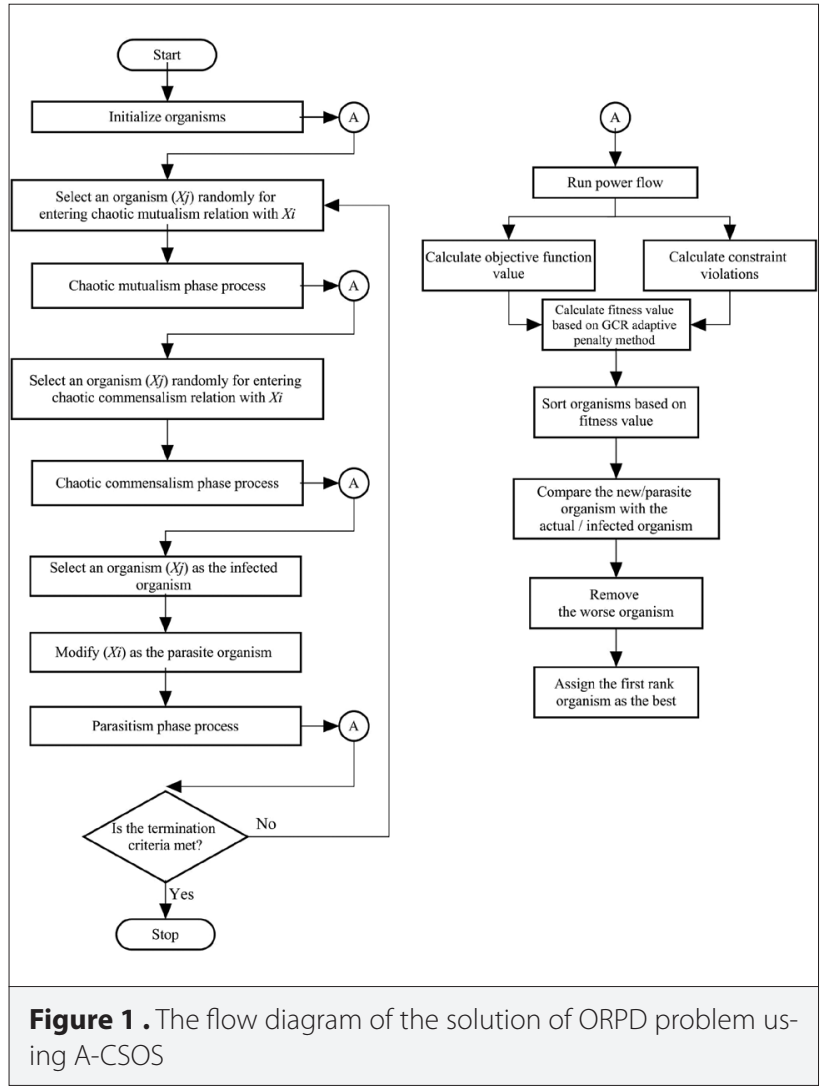

Table 1. The general description of IEEE-30 bus power systems

\section{Parameters}

IEEE 30-bus

\begin{tabular}{ll}
\hline$N_{B}$ & 30 \\
\hline$N_{G e n}$ & 6 \\
\hline$N_{P Q}$ & 24 \\
\hline$N_{T}$ & 4 \\
\hline$N_{C}$ & 9 \\
\hline$N_{\text {reactor }}$ & - \\
\hline$N_{b r}$ & 41 \\
\hline$P_{\text {load (Mw) }}$ & 283.2 \\
\hline$Q_{\text {load (Mvar) }}$ & 126.2 \\
\hline No. of equality contraints & 60 \\
\hline No. of inequality contraints & 125 \\
\hline No. of continuous variable (Case-1 and 3) & 19 \\
\hline No. of discrete variable (Case-1 and 3) & - \\
\hline No. of continuous variable (Case-2 and 4) & 6 \\
\hline No. of discrete variable (Case-2 and 4) & 13 \\
\hline Initial power loss (MW) & 5.5713 \\
\hline Initial TVD (p.u.) & 0.8603 \\
\hline
\end{tabular}


Matpower [25] is used for the test power system data and load flow analysis of the simulations. The permissible maximum iteration is set to 100 . The ecosystem consists of 30 organisms. The algorithm is tested with 30 runs of each test case and the best results are given. The general description and initial condition of test power systems are shown in Table 1 [26].

Table 2. The limits of control variables for IEEE 30-bus

\begin{tabular}{llll}
\hline$V_{G}^{\min }$ & $V_{G}^{\max }$ & $V_{I}^{\min }$ & $V_{I}^{\max }$ \\
\hline 0.95 p.u. & 1.1 p.u. & 0.95 p.u. & 1.1 p.u. \\
\hline$T^{\min }$ & $T^{\max }$ & $Q_{C}^{\min }$ & $Q_{C}^{\max }$ \\
\hline 0.9 p.u. & 1.1 p.u. & 0 Mvar & 5 Mvar \\
\hline
\end{tabular}

The IEEE 30-bus system has 19 control variables which are 6 generator bus voltages, 4 tap ratios of transformers and 9 shunt VAR compensators. The limits of control variables are given in Table 2.

\section{Results of Case-1 using the A-CSOS algorithm}

As mentioned earlier, it is assumed that all control variables are continuous in Case-1. Table 3 presents the optimal value of control variables and the best objective value obtained from 30 test runs for Case-1. The results of the other well-known algorithms are also given in Table 3.

According to Table 3, A-CSOS are able to reduce the Ploss by $19 \%$ with respect to the base case. In comparison with the best result of other algorithms, A-CSOS algorithm gives $0.01621 \mathrm{MW}$ better result.

Table 3. Optimal settings of the control variables for Case-1

\begin{tabular}{|c|c|c|c|c|c|c|c|c|c|c|c|}
\hline Variable & A-CSOS & $\begin{array}{c}\text { HFA } \\
\text { [7] }\end{array}$ & $\begin{array}{c}\text { QOCRO } \\
\text { [12] }\end{array}$ & $\begin{array}{c}\text { PSOGSA } \\
{[6]}\end{array}$ & $\begin{array}{c}\text { BBO } \\
{[5]}\end{array}$ & $\begin{array}{l}\text { DE } \\
{[4]}\end{array}$ & $\begin{array}{c}\text { QOTLBO } \\
{[10]}\end{array}$ & $\begin{array}{c}\text { CLPSO } \\
{[3]}\end{array}$ & $\begin{array}{c}\text { WOA } \\
{[11]}\end{array}$ & $\begin{array}{c}\text { IGSA-CSS } \\
{[15]}\end{array}$ & $\begin{array}{c}\text { MDE } \\
{[14]}\end{array}$ \\
\hline$V_{G 1}$ & 1.10000 & 1.1000 & NR & 1.1000 & 1.1000 & 1.1000 & 1.1000 & 1.1000 & 1.1000 & 1.0813 & 1.07146 \\
\hline$V_{G 2}$ & 1.09430 & 1.0543 & NR & 1.0944 & 1.0944 & 1.0931 & 1.0942 & 1.1000 & 1.0963 & 1.0722 & 1.06222 \\
\hline$V_{G 5}$ & 1.07470 & 1.0751 & NR & 1.0749 & 1.0749 & 1.0736 & 1.0745 & 1.0795 & 1.0789 & 1.0501 & 1.0400 \\
\hline$V_{G 8}$ & 1.07660 & 1.0868 & $N R$ & 1.0767 & 1.0768 & 1.0756 & 1.0765 & 1.1000 & 1.0774 & 1.0502 & 1.0405 \\
\hline$V_{G 11}$ & 1.10000 & 1.1000 & NR & 1.1000 & 1.0999 & 1.1000 & 1.1000 & 1.1000 & 1.0955 & 1.1000 & 1.0804 \\
\hline$V_{G 13}$ & 1.10000 & 1.1000 & NR & 1.1000 & 1.0999 & 1.1000 & 1.0999 & 1.1000 & 1.0929 & 1.0688 & 1.0520 \\
\hline$T_{6-9}$ & 1.04320 & 0.9800 & NR & 1.0452 & 1.0435 & 1.0465 & 1.0664 & 0.9154 & 0.9936 & 1.0800 & 1.0834 \\
\hline$T_{6-10}$ & 0.90000 & 0.9500 & $N R$ & 0.9000 & 0.9011 & 0.9097 & 0.9000 & 0.9000 & 0.9867 & 0.9020 & 0.9000 \\
\hline$T_{4-12}$ & 0.97905 & 0.9701 & NR & 0.9794 & 0.9824 & 0.9867 & 0.9949 & 0.9000 & 1.0214 & 0.9900 & 0.9913 \\
\hline$T_{28-27}$ & 0.96472 & 0.9700 & NR & 0.9651 & 0.9692 & 0.9689 & 0.9714 & 0.9397 & 0.9867 & 0.9760 & 0.9769 \\
\hline$Q_{C 10}$ & 5.00000 & 4.7003 & NR & 5.0000 & 5.0000 & 5.0000 & 5.0000 & 4.9265 & 3.1695 & 0.0000 & 5.0000 \\
\hline$Q_{C 12}$ & 5.00000 & 4.7061 & NR & 5.0000 & 4.9870 & 5.0000 & 5.0000 & 5.0000 & 2.0477 & 0.0000 & 5.0000 \\
\hline$Q_{C 15}$ & 4.80690 & 4.7006 & $N R$ & 5.0000 & 4.9910 & 5.0000 & 5.0000 & 5.0000 & 4.2956 & 3.8000 & 5.0000 \\
\hline$Q_{C 17}$ & 4.99990 & 2.3059 & NR & 5.0000 & 4.9970 & 5.0000 & 5.0000 & 5.0000 & 2.6782 & 4.9000 & 5.0000 \\
\hline$Q_{C 20}$ & 4.03010 & 4.8035 & NR & 3.9792 & 4.9900 & 4.4060 & 4.4500 & 5.0000 & 4.8116 & 3.9500 & 4.0670 \\
\hline$Q_{C 21}$ & 5.00000 & 4.9025 & $N R$ & 5.0000 & 4.9950 & 5.0000 & 5.0000 & 5.0000 & 4.8163 & 5.0000 & 5.0000 \\
\hline$Q_{C 23}$ & 2.51700 & 4.8040 & NR & 2.4583 & 3.8750 & 2.8004 & 2.8300 & 5.0000 & 3.5739 & 2.7500 & 3.1570 \\
\hline$Q_{C 24}$ & 5.00000 & 4.8052 & NR & 5.0000 & 4.9870 & 5.0000 & 5.0000 & 5.0000 & 4.1953 & 5.0000 & 5.0000 \\
\hline$Q_{C 29}$ & 2.19760 & 3.3983 & NR & 2.1865 & 2.9100 & 2.5979 & 2.5600 & 5.0000 & 2.0009 & 2.4000 & 2.9840 \\
\hline BOFV & 4.51279 & 4.5290 & 4.5303 & 4.5309 & 4.5510 & 4.5550 & 4.5594 & 4.5615 & 4.5943 & 4.7660 & 4.8728 \\
\hline TVD & 2.05630 & 1.6250 & 2.0995 & 2.0504 & NR & 1.9589 & 1.9057 & 0.4773 & $N R$ & NR & 0.9051 \\
\hline
\end{tabular}




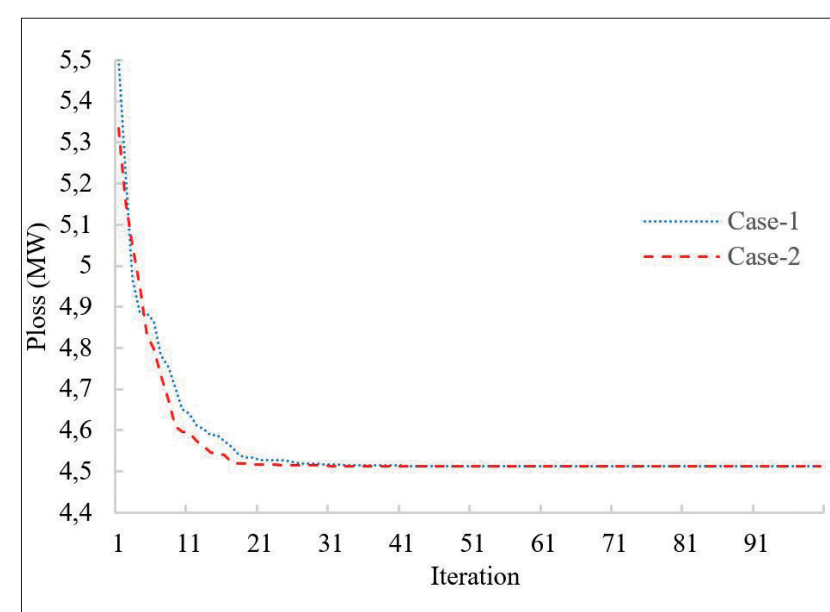

Figure $\mathbf{2}$. The convergence characteristic of A-CSOS for Case-1 and Case-2
The convergence profile of A-CSOS algorithm over 100 iterations for Case-1 is shown in Figure 2. It is seen from the convergence performance of Case-1 optimization in Figure 2, the minimum value convergence obtained by the proposed algorithm is approximately twenty fifth iteration.

\section{Results of Case-2 using the A-CSOS algorithm}

In Case-2 optimization, it is assumed that the output of capacitors and tap ratios are discrete. The minimum value obtained by the A-CSOS algorithm and the other well-known algorithms for Case- 2 analysis and the control parameter values for the best results are presented in Table 4.

Although the optimization problem is more difficult when the control parameters are discrete variables, it is seen that the A-CSOS algorithm achieves much better values than the results obtained with the other algorithms reported in Table 4.

Table 4. Optimal settings of the control variables for Case-2

\begin{tabular}{|c|c|c|c|c|c|c|c|c|c|c|}
\hline Variable & A-csos & GSA [7] & FA [7] & ALO [9] & ABC [7] & GWO [9] & BFOA [7] & BA [9] & PSO [7] & MOCIPSO [16] \\
\hline$V_{G 1}$ & 1.1000 & 1.0999 & 1.1000 & 1.1000 & 1.1000 & 1.1000 & 1.1000 & 1.1000 & 1.1000 & 1.1000 \\
\hline$V_{G 2}$ & 1.0943 & 1.07435 & 1.0644 & 1.0953 & 1.061 & 1.09380 & 1.026 & 1.0940 & 1.1000 & 1.1000 \\
\hline$V_{G 5}$ & 1.0747 & 1.07498 & 1.07455 & 1.0767 & 1.0711 & 1.0737 & 1.0696 & 1.0740 & 1.0850 & 1.1000 \\
\hline$V_{G 8}$ & 1.0761 & 1.07682 & 108690 & 1.0788 & 1.0849 & 1.0797 & 1.1000 & 1.0760 & 1.0838 & 1.1000 \\
\hline$V_{G 11}$ & 1.1000 & 1.0999 & 1.09164 & 1.1000 & 1.1000 & 1.1000 & 1.1000 & 1.1000 & 1.1000 & 1.1000 \\
\hline$V_{G 13}$ & 1.1000 & 1.0999 & 1.0990 & 1.1000 & 1.0665 & 1.0944 & 1.1000 & 1.1000 & 1.1000 & 0.9000 \\
\hline$T_{6-9}$ & 1.0400 & 1.0000 & 1.0000 & 1.0100 & 0.9700 & 0.9800 & 0.9800 & 0.9500 & 1.1000 & 0.9400 \\
\hline$T_{6-10}$ & 0.9000 & 0.9300 & 0.9000 & 0.9900 & 1.0500 & 0.9700 & 0.9400 & 1.0300 & 0.9000 & 1.0800 \\
\hline$T_{4-12}$ & 0.9800 & 0.9800 & 1.0000 & 1.0200 & 0.9900 & 1.0200 & 1.0500 & 0.9900 & 1.0200 & 1.1000 \\
\hline$T_{28-27}$ & 0.9600 & 0.9700 & 0.9700 & 1.0000 & 0.9900 & 0.9900 & 0.9800 & 0.9700 & 0.9900 & 0.9700 \\
\hline$Q_{C 10}$ & 5.0000 & 3.7000 & 3.0000 & 4.0000 & 5.0000 & 2.0000 & 3.1000 & 5.0000 & 1.1000 & 6.0000 \\
\hline$Q_{C 12}$ & 5.0000 & 4.3000 & 4.0000 & 2.0000 & 5.0000 & 5.0000 & 4.6000 & 0.0000 & 0.4000 & 3.0000 \\
\hline$Q_{C 15}$ & 4.8600 & 3.7000 & 3.3000 & 4.0000 & 5.0000 & 4.0000 & 5.0000 & 5.0000 & 0.7000 & 7.0000 \\
\hline$Q_{C 17}$ & 5.0000 & 2.2000 & 3.5000 & 3.0000 & 5.0000 & 4.0000 & 2.1000 & 5.0000 & 5.0000 & 6.0000 \\
\hline$Q_{C 20}$ & 4.0600 & 3.1000 & 3.9000 & 2.0000 & 4.1000 & 4.0000 & 3.7000 & 0.0000 & 4.7000 & 0.0000 \\
\hline$Q_{C 21}$ & 5.0000 & 3.9000 & 3.2000 & 4.0000 & 3.3000 & 0.0000 & 2.3000 & 0.0000 & 1.0000 & 12.000 \\
\hline$Q_{C 23}$ & 2.5300 & 4.2000 & 1.3000 & 3.0000 & 0.9000 & 5.0000 & 1.9000 & 0.0000 & 3.0000 & 3.0000 \\
\hline$Q_{C 24}$ & 5.0000 & 4.4000 & 3.5000 & 5.0000 & 5.0000 & 3.0000 & 2.3000 & 5.0000 & 0.8000 & 7.0000 \\
\hline$Q_{C 29}$ & 1.7700 & 2.0000 & 1.4200 & 5.0000 & 2.4000 & 3.0000 & 0.1000 & 0.0000 & 1.2000 & 3.0000 \\
\hline BOFV & 4.51366 & 4.5400 & 4.5691 & 4.5900 & 4.6022 & 4.6119 & 4.6230 & 4.6280 & 4.6609 & 5.1700 \\
\hline TVD & 2.0708 & 1.9410 & 1.7752 & NR & 0.7378 & NR & 1.5300 & $N R$ & 1.4600 & NR \\
\hline
\end{tabular}




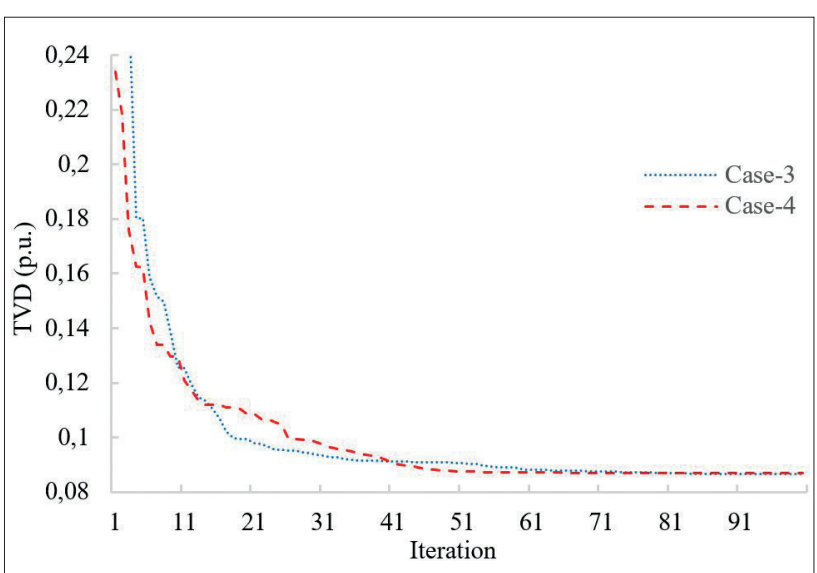

Figure 3. The convergence characteristic of A-CSOS for Case-3 and Case-4
The convergence profile of A-CSOS algorithm for Case-2 optimization is shown in Figure 2. As can be seen in Figure 2, with the contributions of chaos and adaptive penalty approaches, organisms in the ecosystem find the global minimum or near global minimum point in a very short time.

\section{Results of Case-3 using the A-CSOS algorithm}

The TVD minimization denoted Case- 3 adjusts the values of the control parameters so that the voltage magnitudes of the buses can be operated as close as possible to the nominal value specified in the grid code of the countries. It is assumed that all control variables are continuous in Case-3.

Table 5 demonstrates the best TVD value and the value of control parameters within this aim. The TVD value, which is 0.8603 p.u. according to the base case scenario, is reduced to 0.08679 p.u. when optimized with the A-CSOS algorithm. Compared

Table 5. Optimal settings of the control variables for Case-3

\begin{tabular}{|c|c|c|c|c|c|c|c|c|c|c|c|}
\hline Variable & A-CSOS & $\begin{array}{c}\text { IGSA-CSS } \\
{[15]}\end{array}$ & $\begin{array}{c}\text { QOCRO } \\
\text { [12] }\end{array}$ & $\begin{array}{c}\text { PSOGSA } \\
{[6]}\end{array}$ & $\begin{array}{c}\text { MDE } \\
{[14]}\end{array}$ & $\begin{array}{l}\text { DE } \\
{[4]}\end{array}$ & $\begin{array}{c}\text { TLBO } \\
\text { [10] }\end{array}$ & $\begin{array}{c}\text { PSO } \\
{[6]}\end{array}$ & $\begin{array}{c}\text { GSA } \\
\text { [7] }\end{array}$ & $\begin{array}{c}\text { CPVEI HBMO } \\
{[17]}\end{array}$ & $\begin{array}{c}\text { CLPSO } \\
\text { [3] }\end{array}$ \\
\hline$V_{G 1}$ & 1.00940 & 1.0085 & NR & 1.0153 & 1.0000 & 1.0100 & 1.0121 & 1.0264 & 0.9930 & 1.0728 & 1.1000 \\
\hline$V_{G 2}$ & 1.00460 & 1.0057 & NR & 1.0122 & 1.0089 & 0.9918 & 0.9806 & 1.0162 & 0.9552 & 1.0408 & 1.1000 \\
\hline$V_{G 5}$ & 1.01800 & 1.0191 & NR & 1.0185 & 1.0199 & 1.0179 & 1.0207 & 1.0185 & 1.0189 & 1.0379 & 1.0724 \\
\hline$V_{G 8}$ & 1.01100 & 1.0103 & NR & 1.0107 & 1.0000 & 1.0183 & 1.0163 & 0.9987 & 1.0189 & 1.0401 & 1.0764 \\
\hline$V_{G 11}$ & 1.00290 & 1.0184 & NR & 0.9889 & 1.0647 & 1.0114 & 1.0293 & 1.0427 & 1.0120 & 1.0841 & 1.0452 \\
\hline$V_{G 13}$ & 1.01420 & 1.0080 & $N R$ & 1.0083 & 1.0267 & 1.0282 & 1.0323 & 0.9965 & 1.0360 & 1.0220 & 1.1000 \\
\hline$T_{6-9}$ & 1.01760 & 1.0340 & $N R$ & 1.0024 & 1.0852 & 1.0265 & 1.0435 & 1.0598 & 1.0578 & 0.9541 & 1.0177 \\
\hline$T_{6-10}$ & 0.90012 & 0.9000 & $N R$ & 0.9000 & 0.9000 & 0.9038 & 0.9056 & 0.9144 & 1.0500 & 1.1000 & 0.9738 \\
\hline$T_{4-12}$ & 0.99588 & 0.9840 & $N R$ & 0.9791 & 1.0106 & 1.0114 & 1.0195 & 0.958 & 0.9000 & 1.0260 & 1.0244 \\
\hline$T_{28-27}$ & 0.96900 & 0.9780 & $N R$ & 0.9737 & 0.9744 & 0.9635 & 0.9492 & 0.9758 & 1.0500 & 1.0000 & 0.9896 \\
\hline$Q_{C 10}$ & 4.82560 & 5.0000 & $N R$ & 4.3048 & 5.0000 & 4.9420 & 4.8400 & 4.9995 & 0.9660 & 0.0000 & 0.7220 \\
\hline$Q_{C 12}$ & 5.00000 & 5.0000 & NR & 2.3931 & 1.6290 & 1.0885 & 0.6600 & 0.0000 & 4.5000 & 0.0000 & 1.6812 \\
\hline$Q_{C 15}$ & 4.99950 & 5.0000 & $N R$ & 5.0000 & 5.0000 & 4.9985 & 5.0000 & 5.0000 & 2.5000 & 4.3906 & 2.6462 \\
\hline$Q_{C 17}$ & 0.00000 & 0.0000 & $N R$ & 0.0000 & 0.0000 & 0.2393 & 0.0900 & 4.9958 & 1.4000 & 3.3020 & 3.4105 \\
\hline$Q_{C 20}$ & 5.00000 & 5.0000 & $N R$ & 5.0000 & 5.0000 & 4.9958 & 5.0000 & 5.0000 & 4.0000 & 3.5085 & 1.9773 \\
\hline$Q_{C 21}$ & 4.99740 & 5.0000 & $N R$ & 5.0000 & 5.0000 & 4.9075 & 5.0000 & 5.0000 & 3.8000 & 0.0000 & 0.4767 \\
\hline$Q_{C 23}$ & 5.00000 & 5.0000 & $N R$ & 5.0000 & 5.0000 & 4.9863 & 4.9500 & 4.9988 & 2.9000 & 2.4534 & 3.5896 \\
\hline$Q_{C 24}$ & 4.99880 & 5.0000 & $N R$ & 5.0000 & 5.0000 & 4.9663 & 4.9300 & 5.0000 & 2.5000 & 5.0000 & 2.9998 \\
\hline$Q_{C 29}$ & 2.61840 & 4.9500 & NR & 4.1670 & 5.0000 & 2.2325 & 0.2400 & 4.9994 & 3.1000 & 1.8260 & 1.1098 \\
\hline BOFV & 0.08679 & 0.08968 & 0.0899 & 0.0904 & 0.0910 & 0.0911 & 0.0913 & 0.1005 & 0.1180 & 0.1988 & 0.2450 \\
\hline Ploss & 5.8668 & NR & 5.6486 & 5.7344 & 5.9991 & 6.4755 & 7.1859 & 5.5192 & 5.8200 & 4.9948 & 4.6969 \\
\hline
\end{tabular}


with the best results of other algorithms, the A-CSOS algorithm solves the optimization problem better than the other algorithms by $3.22 \%$.

The convergence profile of A-CSOS algorithm for Case-3 optimization is shown in Figure 3.

\section{Results of Case-4 using the A-CSOS algorithm}

In Case-4 optimization, it is assumed that the output of capacitors and tap ratios are discrete. The optimal value of control variables for the obtained minimum TVD value is presented in Table 6. The convergence profile of A-CSOS is shown in Figure 3.

According to Table 6, TVD is to be reduced by $89.89 \%(0.77329$ p.u.) with respect to the base case. In comparison with the best result of declared state-of-art algorithms, A-CSOS algorithm gives $10.39 \%$ (0.01009 p.u.) better result.
It is seen from Figure 3 that A-CSOS finds the minimum and feasible TVD solution approximately in fifth iteration.

\section{Conclusion}

In this paper, SOS algorithm is hybridized with chaos theory and self-adaptive penalty approach in order to design a novel meta-heuristic Adaptive Chaotic Symbiotic Organisms Search Algorithm (A-CSOS) for solving highly nonlinear ORPD problem. The ability of the proposed A-CSOS algorithm is proofed by implementing on both continuos and discrete ORPD problem consisting of active power loss and total voltage deviation minimization in IEEE 30-bus.

According to Case-1 results, it is understood that the A-CSOS algorithm yields $19 \%$ better than the base case and $0.36 \%$ better than the best results of the other algorithms reported. According to Case-2 results, it is seen that the A-CSOS algo-

Table 6. Optimal settings of the control variables for Case-4

\begin{tabular}{|c|c|c|c|c|c|c|c|c|c|}
\hline Variable & A-csos & MALO [8] & HFA [7] & CSA [8] & FA [7] & BA [7] & ALO [7] & $A B C[7]$ & BFO [7] \\
\hline$V_{G 1}$ & 1.0079 & 1.0049 & 1.0035 & 0.9658 & 0.9977 & 1.0186 & 1.0131 & 1.0025 & 0.9500 \\
\hline$V_{G 2}$ & 1.0034 & 0.9504 & 1.0164 & 1.0395 & 1.0217 & 0.9797 & 1.0262 & 1.0162 & 1.0702 \\
\hline$V_{G 5}$ & 1.0181 & 1.0382 & 1.0195 & 1.0198 & 1.0167 & 1.0193 & 1.0194 & 0.9927 & 0.9645 \\
\hline$V_{G 8}$ & 1.0110 & 1.0122 & 1.0182 & 0.9993 & 1.0010 & 1.0475 & 1.0264 & 1.0288 & 1.0258 \\
\hline$V_{G 11}$ & 1.0145 & 1.0406 & 0.9823 & 1.0386 & 1.0481 & 0.9938 & 0.9949 & 1.0647 & 1.0375 \\
\hline$V_{G 13}$ & 1.0090 & 1.0216 & 1.0155 & 1.0494 & 1.0191 & 0.9753 & 0.9732 & 1.0086 & 0.9914 \\
\hline$T_{6-9}$ & 1.0300 & 1.0700 & 0.9900 & 1.0500 & 1.0400 & 0.9800 & 0.9900 & 0.9700 & 0.9800 \\
\hline$T_{6-10}$ & 0.9000 & 0.9100 & 0.9000 & 0.9200 & 0.9000 & 0.9200 & 0.9200 & 1.0300 & 0.9600 \\
\hline$T_{4-12}$ & 0.9800 & 1.0100 & 0.9800 & 1.0500 & 0.9800 & 0.9600 & 0.9500 & 0.9700 & 1.0200 \\
\hline$T_{28-27}$ & 0.9700 & 0.9600 & 0.9600 & 0.9600 & 0.9600 & 0.9700 & 0.9700 & 0.9500 & 0.9900 \\
\hline$Q_{C 10}$ & 5.0000 & 3.8000 & 3.2000 & 0.3900 & 3.6000 & 3.4700 & 4.4000 & 2.5000 & 4.8000 \\
\hline$Q_{C 12}$ & 3.0400 & 4.7600 & 0.5000 & 2.7900 & 1.3000 & 2.4500 & 4.2000 & 0.0000 & 1.3000 \\
\hline$Q_{C 15}$ & 5.0000 & 5.0000 & 4.9000 & 4.7800 & 2.7000 & 3.3700 & 2.6000 & 5.0000 & 4.5000 \\
\hline$Q_{C 17}$ & 0.0000 & 2.2600 & 0.1000 & 5.0000 & 0.9000 & 3.6300 & 1.1000 & 0.0000 & 2.0000 \\
\hline$Q_{C 20}$ & 5.0000 & 4.8400 & 3.8000 & 4.9600 & 4.2000 & 4.3400 & 3.7000 & 5.0000 & 4.3000 \\
\hline$Q_{C 21}$ & 5.0000 & 5.0000 & 5.0000 & 5.0000 & 2.7000 & 3.6200 & 3.4000 & 5.0000 & 3.9000 \\
\hline$Q_{C 23}$ & 5.0000 & 5.0000 & 5.0000 & 5.0000 & 3.0000 & 3.4100 & 3.6000 & 5.0000 & 4.0000 \\
\hline$Q_{C 24}$ & 5.0000 & 5.0000 & 3.9000 & 4.3000 & 1.7000 & 4.0500 & 3.9000 & 4.7000 & 4.5000 \\
\hline$Q_{C 29}$ & 2.8600 & 0.5800 & 1.5000 & 2.7200 & 1.8000 & 2.3500 & 1.9000 & 0.0000 & 3.4000 \\
\hline BOFV & 0.08701 & 0.0971 & 0.0980 & 0.1116 & 0.1157 & 0.1161 & 0.1177 & 0.1350 & 0.1490 \\
\hline TVD & 5.9157 & 5.9020 & 5.7500 & 7.9467 & 6.3400 & 5.6543 & 5.9138 & 5.8800 & 10.570 \\
\hline
\end{tabular}


rithm yields $18.98 \%$ better than the base case and $0.58 \%$ better than the best results of the other latest algorithms. According to Case-3 results, it is understood that the A-CSOS algorithm yields $89.91 \%$ better than the base case and 3.22\% better than the best results of the other state-of-art algorithms. According to Case- 4 results, it is inferred that the A-CSOS algorithm yields $89.89 \%$ better than the base case and $10.39 \%$ better than the best results of the other latest algorithms. Since the ecosystem is sorted in terms of the value of the objective functions and total constraint violation, the proposed algorithm requires more processing and computation time than the standard SOS algorithm. Considering the best results obtained with the proposed algorithm and the elimination of the determining process of penalty coefficients, the additional computation time is acceptable.

The obtained results indicate that the proposed algorithm yields a lower Ploss and TVD value than the best result of the other algorithms. When the results are evaluated, the proposed algorithm yields a lower Ploss and TVD value than the best result of the other algorithms.

Peer-review: Externally peer-reviewed.

Conflict of Interest: The authors have no conflicts of interest to declare.

Financial Disclosure: The authors declared that the study has received no financial support

\section{References}

1. K.Y. Lee, Y.M. Park, J.L. Ortiz, "A united approach to optimal real and reactive power dispatch", IEEE Trans Power Appar Syst, vol. 5, no. 5 , pp. 1147-1153, May, 1985. [CrossRef]

2. J. Carpentier, "Optimal power flows", Electr Power Energy Syst, vol. 1, no. 1, pp. 3-15, Apr, 1979. [CrossRef]

3. K. Mahadevan, P.S. Kannan, "Comprehensive learning particle swarm optimization for reactive power dispatch", Appl Soft Comput, vol. 10, no. 2, pp. 641-652, Mar, 2010. [CrossRef]

4. A.A. Abou El Ela, M.A. Abido, S.R. Spea, "Differential evolution algorithm for optimal reactive power dispatch", Electr Pow Syst Res, vol. 81, no. 2, pp. 458-464, Feb, 2011. [CrossRef]

5. A. Bhattacharya, P.K. Chattopadhyay, "Solution of optimal reactive power flow using biogeography-based optimization", International Journal of Electrical and Computer Engineering, vol. 4, no. 3, pp. 621-629, 2010.

6. J. Radosavljevic, M. Jevtic, M. Milovanovic, "A solution to the ORPD problem and critical analysis of the results", Electr Eng, vol. 100, no. 1, pp 253-265, Mar, 2018. [CrossRef]

7. A. Rajan, T. Malakar, "Optimal reactive power dispatch using hybrid Nelder-Mead simplex based firefly algorithm", Electr Power Energy Syst, vol. 66, pp 9-24, Mar, 2015. [CrossRef]

8. A. Rajan, K. Jeevan, T. Malakar, "Weighted elitism based Ant Lion Optimizer to solve optimum VAr planning problem", Appl Soft Comput, vol. 55, pp. 352-370, Jun, 2017. [CrossRef]

9. S. Mouassa, T. Bouktir, A. Salhi, "Ant lion optimizer for solving optimal reactive power dispatch problem in power systems", Eng Sci Technol Int J, vol. 20, no. 3, pp. 885-895, Jun, 2017. [CrossRef]

10. B. Mandal, P. K.. Roy, "Optimal reactive power dispatch using quasi-oppositional teaching learning based optimization", Electr Power Energy Syst, vol. 53, pp. 123-134, Dec, 2013. [CrossRef]
11. K.b.o. Medani, S. Sayah, A. Bekrar, "Whale optimization algorithm based optimal reactive power dispatch: A case study of the Algerian power system", Electr Power Syst Res, Oct, 2017, unpublished.

12. S. Dutta, S. Paul, P.K. Roy, "Optimal allocation of SVC and TCSC using quasi-oppositional chemical reaction optimization for solving multi-objective ORPD problem", Journal of Electrical Systems and Information Technology, vol. 5, no. 1, pp. 83-98, May, 2018. [CrossRef]

13. B. Shaw, V. Mukherjee, S.P. Ghoshal, Solution of reactive power dispatch of power systems by an opposition-based gravitational search algorithm, Electr Power Energy Syst, vol. 55, pp. 29-40, Feb, 2014. [CrossRef]

14. H. Singh, L. Srivastava, "Modified differential evolution algorithm for multi-objective VAR management", Electr Power Energy Syst, vol. 55, pp. 731-740, Feb, 2014. [CrossRef]

15. G. Chen, L. Liu, Z. Zhang, S. Huang, “Optimal reactive power dispatch by improved GSA-based algorithm with the novel strategies to handle constraints", Appl Soft Comput, vol. 50, pp. 58-70, Jan, 2017. [CrossRef]

16. G. Chen, L. Liu, P. Song, Y. Du, "Chaotic improved PSO-based multi-objective optimization for minimization of power losses and Lindex in power systems", Energy Convers Manage, vol. 86, pp. 548-560, Oct, 2014. [CrossRef]

17. A. Ghasemi, K. Valipour, A. Tohidi, "Multi objective optimal reactive power dispatch using a new multi objective strategy", Electr Power Energy Syst, vol. 57, pp. 318-334, May, 2014. [CrossRef]

18. M. Mehdinejad, B. Mohammadi-Ivatloo, R. Dadashzadeh-Bonab, K. Zare, "Solution of optimal reactive power dispatch of power systems using hybrid particle swarm optimization and imperialist competitive algorithms", Electr Power Energy Syst, vol. 83, pp. 104-116, Dec, 2016. [CrossRef]

19. M.Y. Cheng, D. Prayogo, "Symbiotic Organisms Search: A new metaheuristic optimization algorithm", Computers \& Structures, vol. 139, pp. 98-112, Jul, 2014. [CrossRef]

20. U. Guvenc, S. Duman, M.K. Dosoglu, et al., "Application of Symbiotic Organisms Search Algorithm to solve various economic load dispatch problems", 2016 International Symposium on INnovations in Intelligent SysTems and Applications, Sinaia, Romania, 2016. [CrossRef]

21. B. Das, V. Mukherjee, D. Das, “DG placement in radial distribution network by symbiotic organisms search algorithm for real power loss minimization", Applied Soft Computing, vol. 49, pp. 920-936, Dec, 2016. [CrossRef]

22. D.C. Secui, "A modified Symbiotic Organisms Search algorithm for large scale economic dispatch problem with valve-point effects", Energy, vol. 113, pp. 366-384, Oct, 2016. [CrossRef]

23. A. Saha, A.K. Chakraborty, P. Das, "Quasi-reflection based symbiotic organisms search algorithm for solving static optimal power flow problem", Scientia Iranica, Feb, 2018.

24. T.P. Runarsson, X. Yao, "Constrained evolutionary optimization," in Evolutionary Optimization: International Series in Operations Research\&Management Science, Boston, MA: Springer, 2003, vol. 48, pp. 87-113. [CrossRef]

25. R.D. Zimmerman, C.E. Murillo-Sanchez, R.J. Thomas, "MATPOWER: Steady-State Operations, Planning and Analysis Tools for Power Systems Research and Education", IEEE Trans Power Syst, vol. 26, no. 1, pp. 12-19, Feb, 2011. [CrossRef]

26. The IEEE 30-bus test system. (2017, Mar 15). Retrieved from http:// www.ee.washington.edu/research/pstca/pf30/pgtca30bus.htm 
Enes Yalçın received the B.Sc degree in Electrical and Electronics Engineering from Kırıkkale University in 2006 and M.Sc degree in Electrical and Electronics Engineering from Kırıkkale University in 2010. He is currently a Technical Inspector in TEIAŞ and Ph.D student at Gazi University. His research interests include power system optimization, optimal power flow, grid integration of renewable energy sources and transmission system planning.

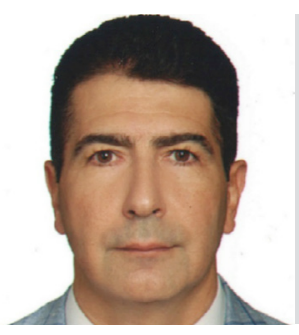

Müslüm Cengiz Taplamacıoğlu graduated from Department of Electrical and Electronics Engineering, Gazi University. He received the degrees of M.Sc. in Industrial Engineering from Gazi University and in Electrical and Electronics Engineering from Middle East Technical University and received the degree of Ph.D. in Electrical, Electronics and System Engineering from University of Wales (Cardiff, UK). He has been a full time Professor of the Electrical and Electronics Engineering since 2000. He is currently working as a Professor at Gazi University. His research areas consist of high voltage engineering, optimization of power system operation and control problems, renewable energy source integration problems, electrical field computation, power systems and protection devices.

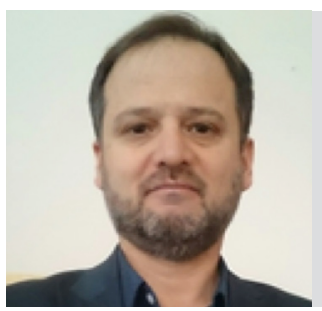

Ertuğrul Çam received the B.Sc degree in Electrical and Electronics Engineering from Dokuz Eylul University in 1996, the M.Sc degree in Electrical and Electronics Engineering from Ege University in 1999 and the Ph.D degree in Mechanical Engineering from Kırıkkale University in 2004. He is currently working as a Professor at Kırıkkale University. His research areas consist of power system control, renewable energy sources, fuzzy logic. 